\title{
Loitering behaviour detection of boats at sea
}

Conference or Workshop Item

Accepted Version

Patino, L. and Ferryman, J. (2017) Loitering behaviour detection of boats at sea. In: IEEE Conference on Computer Vision and Pattern Recognition Workshops (CVPRW), 26 July 2017, Honolulu, HI, USA. Available at http://centaur.reading.ac.uk/75243/

It is advisable to refer to the publisher's version if you intend to cite from the work. See Guidance on citing.

Published version at: http://ieeexplore.ieee.org/document/8015003

All outputs in CentAUR are protected by Intellectual Property Rights law, including copyright law. Copyright and IPR is retained by the creators or other copyright holders. Terms and conditions for use of this material are defined in the End User Agreement. 


\section{CentAUR}

Central Archive at the University of Reading

Reading's research outputs online 


\title{
Loitering behaviour detection of boats at sea
}

\author{
Luis Patino and James Ferryman \\ University of Reading, Computational Vision Group \\ Whiteknights, Reading RG6 6AY, United Kingdom \\ \{j.l.patinovilchis, j.m.ferryman\}@reading.ac.uk
}

\begin{abstract}
We present in this paper a technique for Loitering detection based on the analysis of activity zones of the monitored area. Activity zones are learnt online employing a soft computing-based algorithm which takes as input the trajectory of object mobiles appearing on the scene. Statistical properties on zone occupancy and transition between zones makes it possible to discover abnormalities without the need to learn abnormal models beforehand. We have applied this approch to the PETS2017 IPATCH dataset and addressed the challenge on detecting skiff boats loitering around a protected ship, which eventually is attacked by the skiffs. Our results show that we can detect the suspicious behaviour on time to trigger an early warning.
\end{abstract}

\section{Introduction}

The beginning of the 21st century has seen a resurgence of maritime piracy. According to the International Maritime Bureau's (IMB) Piracy Reporting Center, piracy has been rising steadly for years [22]. Some regions in the Gulf of Aden, West Africa, South East Asia and South America have turned into dangerous places for commercial ships. It is of utmost importance for ships in high risk areas to detect piracy threats as early as possible so that the ship master can initiate countermeasures while they are still effective.

Several events are useful indicators of an early warning of a maritime threat. We can cite among them: 'boat speeding up', the sudden acceleration of the mobile object; 'boat loitering', the detected object stands/moves slowly in the same area; 'boat with anomalous direction', an object has a trajectory deviating from normal paths. While some other works have addressed some of these events [4, 7, 21, 23], loitering remains still to be characterised/exploited in the maritime domain.

In this work we focus on the detection of Loitering events, as set in the PETS2017 challenge on the maritime IPATCH dataset. The proposed approach works by first detecting Activity zones. Activity zones are learnt on-line employing a soft computing-based algorithm which takes as input the trajectory of detected mobile objects. Statistical properties on zone occupancy and transition makes it possible to discover abnormalities without the need to learn abnormal models beforehand.

The remainder of this paper is organised as follows. The next section gives a short overview of the related work. The general system description is presented in Section 3. In Section 4 , it is explained how the boat loitering behaviour is addressed. Section 5 presents the main results and evaluation. Finally, Section 6 draws the main conclusions and includes our future work.

\section{Related work}

Maritime surveillance has become a subject of utmost importance as the beginning of the 21 st century has seen a resurgence of maritime piracy. According to the International Maritime Bureau's (IMB) Piracy Reporting Centre, piracy has been rising steadily for years [22]. Some regions in the Gulf of Aden, West Africa, South East Asia and South America have turned into dangerous places for commercial ships.

Different threats have been searched in the literature. Most works are based on the analysis of vessel trajectory provided by AIS and/or radar. Clustering methods have been popular to detect ship traffic-related abnormalities (deviating from normal routes; travelling in an anomalous direction; travelling in a sea lane but at an anomalously high speed; crossing the main sea lane at an anomalous location; stopping in a prohibited anchoring location) $[4,7,21,23,20]$ or learning contextual areas at sea, such as fishing areas or high piracy risk areas [5]. Detecting piracy attacks has been the subject of some specific works. Garcia, et al. [22] looks to build a reliable surveillance picture based on matching boat tracking information with an ontology and using Belief Theory [24]. The boat features analysed for the threat are speed, direction, type and flag state. Bouejla et al. [3] have addressed piracy attacks against offshore oil infrastructure (oil platforms or oil tankers); the parameters employed to characterise the asset in danger include 
the threat itself (the type of ship used by the attackers, its speed and their weapons) and the environmental conditions (the time of day, visibility, sea state, etc.). Lane et al. [13] have employed Bayesian networks to detect threatening behaviours such as 'close approach' or 'unexpected activity' based on location, speed and heading of the suspicious boat. Dabrowski, et al. [6] classify vessels as pirate vessels, fishing vessels or transport vessels based on the tracked trajectory of the vessel.

Loitering itself has been acknowledged to be important for detection of a maritime threat [19]. Loitering refers to a boat spending abnormally long time on the same area or performing slow movements around a bigger area but still without displacing itself a significant distance. This behaviour has been studied extensively in its land-case counterpart as loitering is a suspicious behavior that might lead to abnormal situations, such as pickpocketing, attack to a person or to a valuable asset [2, 11, 9, 17].

However, Loitering has generally been addressed as detecting an object on the scene for a period of time that exceeds a given threshold [15, 14, 12]. This means that manual adjustments on setting the threshold have to be done according to the dynamics of the scene under scrutiny. Instead, we propose in this work to learn statistical properties on zone occupancy that will allow automatic detection of Loitering behaviour without the need of setting temporal thresholds beforehand. Our system would then start by extracting the activity zones of the scene. Such zones would be analysed at several resolutions to allow for detection of Loitering on small or larger areas. Our contribution to the literature is thus a methodology for loitering detection applied to the maritime domain, which is based on learning statistical properties on zone occupancy without the need of setting thresholds.

\section{System description}

In this work we propose an extension to the behaviour analysis framework given in [18]; which is based on Semantic modelling and trajectory analysis. In such framework, the system can learn activity zones (context zones) that characterise the scene dynamics. We extend that system by extracting temporal statistics of object mobiles at each zone to detect the abnormal behaviour of an object Loitering.

The system is composed of three modules applied sequentially. In the first module, Tracklet calculation process (Extraction of points of interest), we study the trajectory speed and direction variations in order to distinguish between the object mobile in a stationary state (stop points) or moving state; between the object mobile in a straight direction or changing direction. The second module is a Zone learning module where we aim here to automatically discover activity zones of the scene. In a first step, points of interest obtained from the previous calculation process are clustered together to obtain a first set of zones. In a second step zones are refined with a zone merging procedure. Indeed, It is possible that some discovered activity zones are partially overlapping. When this is the case, both regions are most certainly part of a bigger activity zone. Our algorithm will attempt to merge those overlapping activity zones. The third module is the Event Detection module: Statistical confidence levels are applied on zone occupancy measures to discover object mobiles spending suspiciously long periods on a given zone. Note that the system requires a detection and tracking algorithm that delivers trajectories of each mobile object on the scene. The hypothesis is thus that such a system is operational. In this work we employ for our study the trajectories made available on the PETS IPATCH dataset.

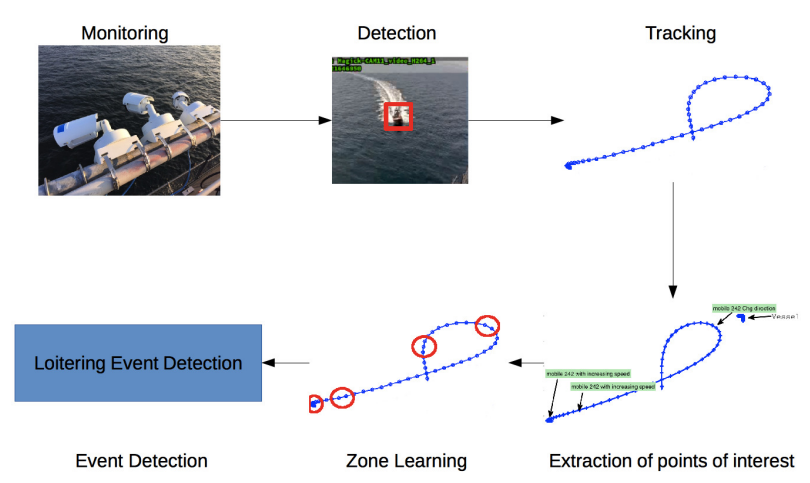

Figure 1. Processing chain for the proposed approach. Note that this work includes only modules displayed in the lower raw. A detection and tracking algorithm is assumed but not considered in this work.

\section{Boat Behaviour Analysis}

Loitering detection is based on the analysis of activity zones of the monitored area. Statistical properties on zone occupancy and transition between zones makes it possible to discover abnormalities without the need to learn abnormal models beforehand. In our work, activity zones are learnt on-line employing as input the trajectory of object mobiles appearing on the scene. Two essential procedures in our work are thus the trajectory processing and the discovery (learning) of activity zones, which are explained next.

\subsection{Trajectory Processing}

In order to detect boats abnormally staying long periods of time around the protected ship, we analyse the monitored boats speed profile. We aim to extract indicators of movement such as speeding up or staying still in the vicinity of the ship in order to distinguish loitering. We analyse the 
boat direction to complement with points of interest indicating a direction change of the boat, and if the boat still remains in the vicinity of the ship this may be a further indication of loitering. Figure 2 shows an example of extracted speed points of interest.

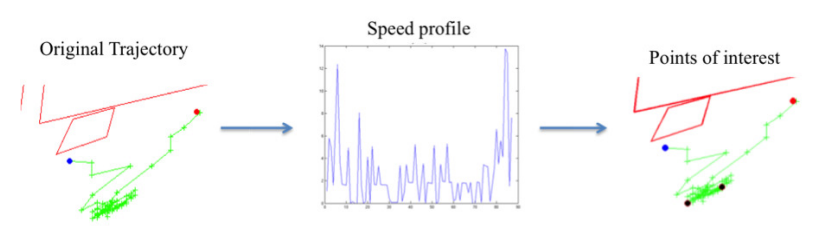

Figure 2. speed-related points of interest are extracted from the analysis of the mobile trajectory

Each mobile trajectory is defined as the set of points $\left[x_{j}(t), y_{j}(t)\right]$ corresponding to their position on the ground on the t-th frame. The instantaneous speed for that mobile at point $\left[x_{j}(t), y_{j}(t)\right]$ is then $v(t)=\left(\dot{x}(t)^{2}+\dot{y}(t)^{2}\right)^{\frac{1}{2}}$, and the direction $\theta$ that the mobile takes at that point is $\theta(t)=\arctan (\dot{y}(t) / \dot{x}(t))$.

Each of these two time series is analysed in the frame of a multiresolution analysis [16] with a Daubichis Haar smoothing function, $\rho_{2^{s}}(t)=\rho\left(2^{s} t\right)$, to be dilated at different scales $s$.

In this frame, the approximation $A$ of $v(t)$ by $\rho$ is such that $A_{s-1}(v)=\int v(t) \rho\left(2^{s-1} t-b\right) d t$ is a broader approximation of $A_{s} v$ and correspondly for $A_{s-1}(\theta)$ and $A_{s} \theta$. The analysis is performed through six dyadic scales. Speed changing points and direction changing points correspond to those important discontinuities which remain present across scales.

\subsection{Zone Learning}

Activity zones are computed having inputs of track speed and direction changing points calculated as explained in Section 4.1. These points are first clustered by a fast partitioning algorithm (the Leader algorithm [8, 10]), allowing to quickly create an initial set of zones $\mathrm{Zn}$. In a second step the partition is corrected leading to the final activity zones. To correct the initial partition, different relationships between initial zones $\mathrm{Zn}$ are taken into account. For this step, soft computing techniques are employed.

\subsubsection{Soft computing relation clustering}

Let us consider two different fuzzy binary relations, $R I$ and $R 2$, linking different sets $X, Y$, and $Z$ :

- $\mathrm{R} 1=\mathrm{x}$ is relevant to $\mathrm{y}$

- $\mathrm{R} 2=\mathrm{y}$ is relevant to $\mathrm{z}$
It is then possible to find to which extent $x$ is relevant to $z$ by employing the extention principle (noted $R=R 1 o R 2$ ):

$$
\mu_{R=R 1 \circ R 2}(x, z)=\max _{y} \min \left[\mu_{R 1}(x, y), \mu_{R 2}(y, z)\right]
$$

$\mu_{R 1}$ and $\mu_{R 2}$ being the membership functions defining $R 1$ and $R 2$. The resulting relation, $R$, is symmetric, $R(x, y)=R(y, x)$, reflexive $R(x, x)=1 ; R$ is also a transitive relation. $R(x, y)$ is a transitive relation if $\exists z \in$ $X, z \in Y / R(x, y) \geqslant \max _{z} \min [R(x, z), R(z, y)]$. $R$ can be made furthermore closure transitive following the next steps

Step1. $R^{\prime}=R \cup(R \circ R)$

Step2. If $R^{\prime} \neq R$, make $R=R^{\prime}$ and go to step1

Step3. $R=R^{\prime}$ Stop.

$\mathrm{R}$ is the transitive closure where

$$
R \circ R(x, y)=\max _{z} \min (R(x, z), R(z, y))
$$

\subsubsection{Initial zone learning partition}

For the first step, the clustering Leader algorithm is employed. It has the advantage of working on-line without needing to specify the number of clusters in advance. In this method, it is assumed that a threshold $T$ is given. The algorithm constructs a partition of the input space (defining a set of clusters) and a leading representative for each cluster, so that every object in a cluster is within a distance $T$ of the leading object. The first pattern is assigned to a cluster. Then the next pattern is assigned to an existing cluster or to a new cluster depending on the distance between the pattern and the cluster leading representative. The process is repeated until all patterns are assigned to clusters.

In this application, when a point is designed as cluster leader (or leading representative), $L$, the cluster influential zone, $Z n$, is defined by a radial basis function (RBF) centered at the position $L$; and the membership of a new point $p(x, y)$ to that zone is given by:

$$
Z n(L, p)=\phi(L, p)=\exp \left(-\|p-L\|^{2} T^{2}\right)
$$

The RBF function has a maximum of 1 when its input is $p=L$ and thus acts as a similarity detector with decreasing values outputted whenever $p$ strides away from $L$. An object element will be included into a cluster if $Z n(L, p) \geqslant 0.5$. The cluster receptive field (hypersphere) is controlled by the learnt parameter $T$. In this work we employ the same settings as in [18] 


\subsubsection{Final zone learning partition}

The final activity areas are found by merging similar initial zones $\mathrm{Zn}$. They are established by the fulfilment of different relations between zones $\mathrm{Zn}$. This procedure is achieved by setting soft computing relationships between zones.

The first relation indicates if zone $Z n_{i}$ overlaps zone $Z n_{j}$. This relation is defined as follows:

$R 1_{i j}$ : Zone $Z n_{i}$ overlaps Zone $Z n_{j}$

$$
R 1_{i j}=\sum_{k=1}^{3}\left[\sum_{p(x, y) \in\left(X_{i k}, Y_{i k}\right)} Z n_{j}\left(L_{j}, p(x, y)\right)\right]
$$

and $\left(X_{i k}, Y_{i k}\right)=\left\{\frac{(k+1)}{3} T \cos (\theta)+L_{i}\right\}$ with $\theta=0, \ldots, \frac{\pi}{8}, \ldots, 2 \pi$

That is, points $(x, y) \in\left(X_{i k}, Y_{i k}\right)$ belonging to $Z n_{i}$ centered at $L_{i}$ are tested to verify the overlap/similarity between $Z n_{i}$ and $Z n_{j}$.

Similar relations that we have introduced are as follows:

$R 2_{i j}^{t}$ : zone $Z n_{i}$ and zone $Z n_{j}$ are destination zones for mobiles departing from the the same activity zone $Z n_{k}$

$R 3_{i j}^{t}$ : zone $Z n_{i}$ and zone $Z n_{j}$ are origin zones for mobiles arriving at the the same activity zone $Z n_{k}$

$R 4_{i j}^{t}$ : zone $Z n_{i}$ and zone $Z n_{j}$ have about the same number of detected mobiles stopping at the zone

$R 5_{i j}^{t}$ : zone $Z n_{i}$ and zone $Z n_{j}$ have about the same mobile interaction time. The mobile interaction time is the mean time a mobile spends in that zone.

All relations can be aggregated employing a soft computing aggregation operator such as

$R=R 1 \cap R 2 \cap R 3 \cap R 4 \cap R 5=$ $\max (0, R 1+R 2+R 3+R 4+R 5-4)$ and made transitive with the formulae given in Equation 1.

$R$ then indicates the strength of the similarity between $Z n_{i}$ and $Z n_{j}$. If we define a discrimination level $\alpha$ in the closed interval $[0,1]$, an $\alpha-c u t$ can be defined such that

$$
R^{\alpha}(x, y)=1 \Leftrightarrow R(x, y) \geqslant \alpha
$$

From the classification point of view, $R^{\alpha}$ induces a new partition $\pi^{\alpha}$ with a new set of clusters $\pi^{\alpha}=$ $\left\{A Z n_{1}^{\alpha}, \cdots, A Z n_{k}^{\alpha}, \cdots, A Z n_{\left|\pi^{\alpha}\right|}^{\alpha}\right\}$ such that cluster $A Z n_{k}{ }^{\alpha}$ is made of all initial zones $Z n_{i}$ which up to the alpha level fulfil the relations set above and can thus be merged to form the final activity zone $A Z n_{k}^{\alpha}$.

\subsection{Boat loitering detection}

Loitering detection corresponds essentially, in this work, to measuring the amount of time boats may stay stationary on a given area (activity zone), and determine whether it is significantly different from the normal amount of time boats usually stay around the protected ship. More specifically, we want to establish an statistical measure indicating a boat
'Unusually (abnormally) stays long at any zone' around the protected ship. The decision on abnormality is based on the calculation of statistical confidence levels.

This statistics-based methodology is based on the idea that 'normal' data objects follow a generating mechanism, e.g. some given statistical process (distribution model). 'Abnormal' objects deviate from this generating mechanism.

Such statistical measure is mathematically defined with the following equation:

$$
C L=1.96 *\left(\frac{\sigma}{\sqrt{n}}\right)
$$

where $\sigma$ is the standard deviation of the measured parameter, $\mu$, and $n$ is the number of observations of such parameter. The confidence level can be interpreted as having $95 \%$ confidence that the true value of $\mu$ lies between $[\bar{\mu}-C L, \bar{\mu}+C L]$. Such statistical bounds are employed as a reference to decide whether a measurement could be considered suspicious by comparing whether it lies inside or outside such statistical bounds. The abnormality of a given measurement is graded depending on how far it lies from these statistical bounds.

It is assumed in this work a normal distribution of the data for abnormality detection, that is, it is assumed 'normal activities' ('staying in a zone', 'undertaken path frequencies') can be characterised by a mean value and symmetrical tolerance intervals around it. Furthermore, the central limit theorem states that even if the population distribution is unknown, the sampling distribution of the mean will be approximately normally distributed if the sample size is large.

Let stayinZntime $\left(\operatorname{tr}_{j}, A Z n_{k}^{\alpha}\right)$ be the time a mobile spends in the given activity zone $A Z n_{k}^{\alpha}$. A mobile trajectory is considered to suspiciously stay long in that activity zone at resolution $\alpha$ if its stayinZntime measure lies outside the confidence values; grading the abnormality by calculating the ratio

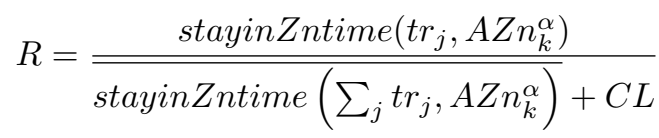

\section{Experimental Results}

We have addressed the challenge set in PETS2017 [1] regarding the Loitering behaviour recognition of a boat in the vicinity of a protected ship. As such, we have evaluated the two sequences from the PETS dataset containing this behaviour. The two test sequences are namely Sc3_Tk1 and Sc3_Tk3. Scenario 3 corresponds in the dataset to a slow approach from two skiffs to a protected ship equipped with 


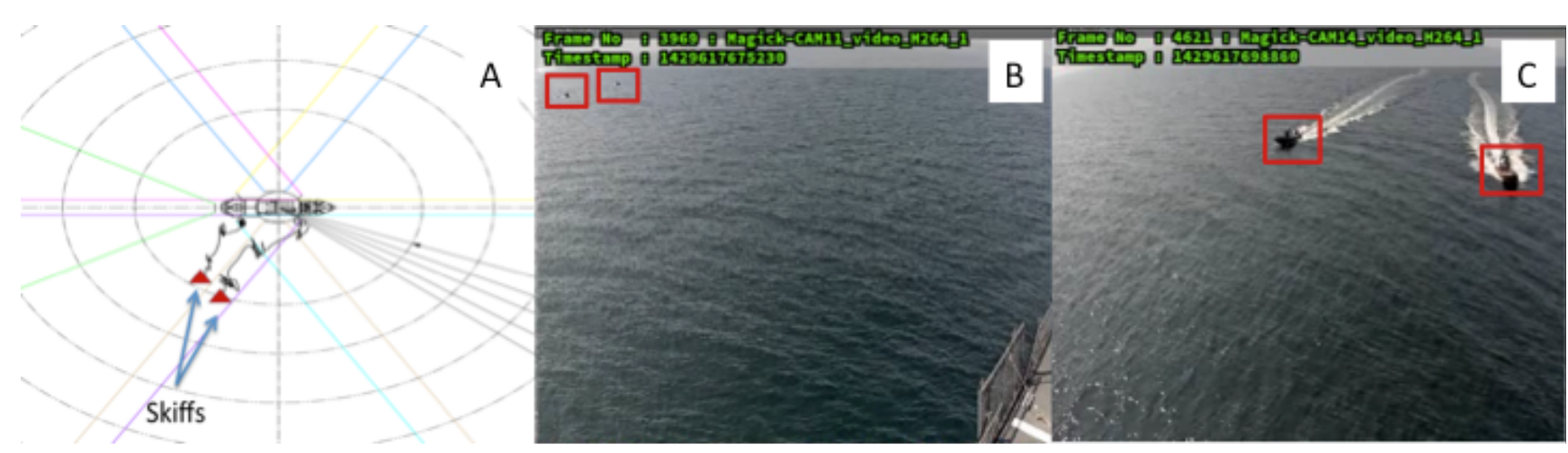

Figure 3. Sc3_Tk1 of the IPATCH-PETS dataset. A) The schematic representation of the scenario indicates two skiffs loitering before speeding up to attack a vessel. B) Two skiffs observed stationary (Loitering) by one of the ship cameras. C) The two skiffs observed speeding up for an attack against the vessel.

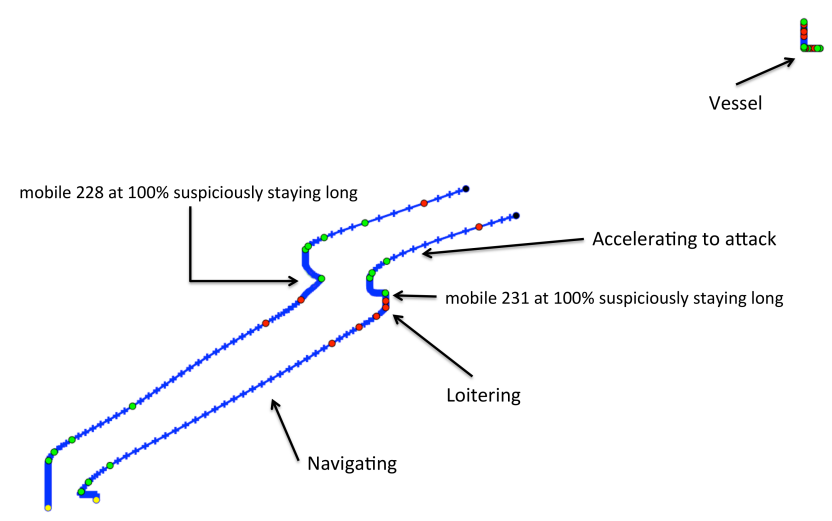

Figure 4. Sc3_Tk1 of the PETS-IPATCH dataset. The two skiffs have a loitering period before performing an attack to the ship. Red points indicate boat decreasing speed. Green points indicate boat increasing speed.

cameras to monitor the close range space. The skiffs first remain long periods stationary, simulating being fishermen, before speeding up to attack the ship. These two sequences are appropriate for test as they contain long periods of stationary not far from the protected ship that can be considered as loitering activity. Figure 3 shows the schematic interpretation of scenario 3 and provides some examples of the skiffs captured by one of the ship cameras, first in stationary state (Loitering) and then approaching the ship at high speed to perform an attack.

Our results to detect the Loitering state are summarised in Table 1 respectively for scenarios Sc3_Tk1 and Sc3_Tk3. Visual validation is used to verify if the result is a True Positive (TP), False Positive (FP), True Negative (TN) or False Negative (FN). The real trajectories for the corresponding sequences are shown in Figures 4 and 5 for each of the processed sequences together with the detected loitering event. Note that although in the figures the loitering event is de-

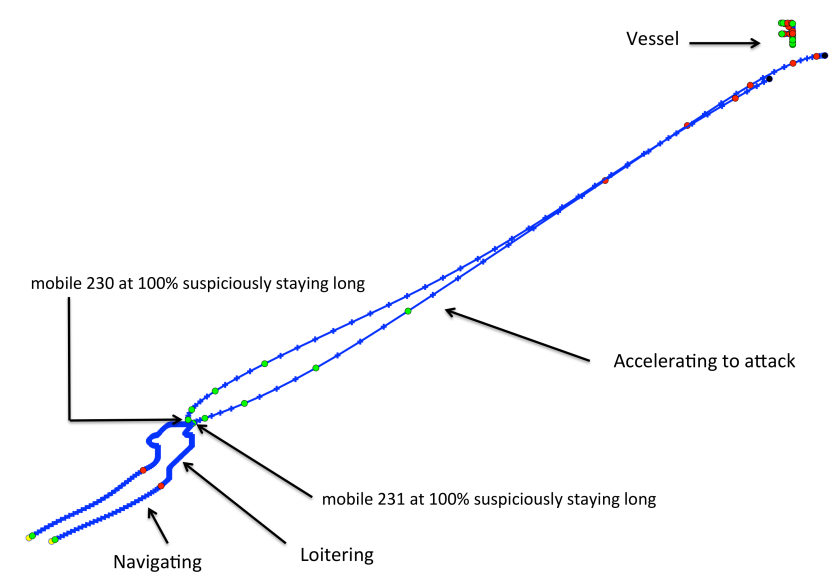

Figure 5. Sc3_Tk3of the PETS-IPATCH dataset. The two skiffs have a loitering period before strongly speeding up to attack the ship. Red points indicate boat decreasing speed. Green points indicate boat increasing speed.

picted at the end of the stationary state, the system assessing the situation around the ship continously and giving the likelihood of loitering as calculated from equations 6 and 7. In Table 1, it can be observed that loitering events are given with gradual levels of warning. It could be argued that possibly when the threat or warning level is low (probably less than 50\%) no event warning should be given and as such we have marked produced events under $50 \%$ confidence as FP and could probably not be forwarded to the end user. The remaining of the produced events are marked as definite True Positives as they carry a high level of confidence and can be visually validated.

\section{Conclusions}

In this work we have addressed the challenge set on PETS 2017 regarding loitering detection of boats around 


\begin{tabular}{|l|l|l|l|l|}
\hline Sequence & trackid & eventhhmmss & event \\
\hline Sc3_Tk1 & Boat 2 & $21 / 04 / 1512: 00: 00$ & mobile 228 at 28\% suspicsously staying long \\
\hline Sc3_Tk1 & Boat 1 & $21 / 04 / 1512: 00: 00$ & mobile 227 at 28\% suspicsously staying long \\
\hline Sc3_Tk1 & Boat 1 & $21 / 04 / 1512: 00: 40$ & mobile 227 at 87\% suspicsously staying long \\
\hline Sc3_Tk1 & Boat 2 & $21 / 04 / 1512: 00: 40$ & mobile 228 at 87\% suspicsously staying long \\
\hline Sc3_Tk1 & Boat 1 & $21 / 04 / 1512: 01: 20$ & mobile 227 at 100\% suspicsously staying long \\
\hline Sc3_Tk1 & Boat 2 & $21 / 04 / 1512: 01: 20$ & mobile 228 at 100\% suspicsously staying long \\
\hline Sc3_Tk1 & Boat 1 & $21 / 04 / 1512: 01: 36$ & mobile 227 at 100\% suspicsously staying long \\
\hline Sc3_Tk1 & Boat 2 & $21 / 04 / 1512: 01: 36$ & mobile 228 at 100\% suspicsously staying long \\
\hline Sc3_Tk3 & Boat 1 & $21 / 04 / 1512: 16: 45$ & mobile 230 at 28\% suspicsously staying long \\
\hline Sc3_Tk3 & Boat 2 & $21 / 04 / 1512: 16: 45$ & mobile 231 at 28\% suspicsously staying long \\
\hline Sc3_Tk3 & Boat 1 & $21 / 04 / 1512: 17: 17$ & mobile 230 at 75\% suspicsously staying long \\
\hline Sc3_Tk3 & Boat 2 & $21 / 04 / 1512: 17: 17$ & mobile 231 at 75\% suspicsously staying long \\
\hline Sc3_Tk3 & Boat 1 & $21 / 04 / 1512: 17: 57$ & mobile 230 at 100\% suspicsously staying long \\
\hline Sc3_Tk3 & Boat 2 & $21 / 04 / 1512: 17: 57$ & mobile 231 at 100\% suspicsously staying long \\
\hline Sc3_Tk3 & Boat 1 & $21 / 04 / 1512: 18: 37$ & mobile 230 at 100\% suspicsously staying long \\
\hline Sc3_Tk3 & Boat 2 & $21 / 04 / 1512: 18: 37$ & mobile 231 at 100\% suspicsously staying long \\
\hline
\end{tabular}

Table 1. Recognised behaviours in the PETS-IPATCH dataset.

a protected ship. The approach is based on the analysis of real trajectories provided in the IPATCH dataset. First, activity zones are learnt online employing a soft computingbased algorithm which takes as input the trajectory 'change of speed' or 'change of direction' points, extracted from the object trajectories. Statistical properties on zone occupancy will allow automatic detection of Loitering behaviour. Contrasting with state of the art algorithms, we can perform loitering detection without the need of setting temporal thresholds beforehand. The results obtained on the PETS2017 IPATCH dataset are encouraging and to our knowledge this is a pioneering work on loitering detection on the maritime domain. Our future work will include the analysis of tracks fusing different sensors such as Radar, AIS and camera tracking.

\section{Acknowledgement}

This project has received funding from the European Union's Seventh Framework Programme for research, technological development and demonstration under grant agreement no. 607567.

\section{References}

[1] 1st Joint BMTT-PETS Workshop on Tracking and Surveillance, https://motchallenge.net/workshops/bmtt-pets2017/. 4

[2] N. D. Bird, O. Masoud, N. P. Papanikolopoulos, and A. Isaacs. Detection of loitering individuals in public transportation areas. IEEE Transactions on Intelligent Transportation Systems, 6(2):167-177, June 2005. 2

[3] A. Bouejla, X. Chaze, F. Guarnieri, and A. Napoli. A bayesian network to manage risks of maritime piracy against offshore oil fields. Safety Science, 68:222 - 230, 2014. 1

[4] F. Castaldo, F. A. N. Palmieri, V. Bastani, L. Marcenaro, and C. Regazzoni. Abnormal vessel behavior detection in port areas based on dynamic bayesian networks. In Information Fusion (FUSION), 2014 17th International Conference on, pages $1-7$, July 2014. 1

[5] J. J. Dabrowski and J. P. de Villiers. Maritime piracy situation modelling with dynamic bayesian networks. Information Fusion, 23:116 - 130, 2015. 1
[6] J. J. Dabrowski and J. P. de Villiers. A unified model for context-based behavioural modelling and classification. Expert Systems with Applications, 42(19):6738-6757, 2015. 2

[7] A. Dahlbom and L. Niklasson. Trajectory clustering for coastal surveillance. In Information Fusion, 2007 10th International Conference on, pages 1-8. IEEE, 2007. 1

[8] R. Duda, P. Hart, and D. Stork. Pattern Classification and Scene Analysis. John Wiley \& Sons, Inc., New York, 1995. 3

[9] H. F. Gómez A., R. M. Tomás, S. A. Tapia, A. F. Caballero, S. Ratté, A. G. Eras, and P. L. González. Identification of Loitering Human Behaviour in Video Surveillance Environments, pages 516-525. Springer International Publishing, Cham, 2015. 2

[10] J. A. Hartigan. Clustering algorithms. John Wiley \& Sons, Inc., New York, 1975. 3

[11] S.-R. Ke, H. L. U. Thuc, Y.-J. Lee, J.-N. Hwang, J.-H. Yoo, and K.-H. Choi. A review on video-based human activity recognition. Computers, 2(2):88-131, 2013. 2

[12] Y. Kim, K. Kim, and Y.-S. Kim. Loitering event detection scheme using partial trajectory features. International Information Institute (Tokyo).Information, 19(9):3801-3808, 09 2016. Copyright - Copyright International Information Institute Sep 2016; Document feature - Equations; Diagrams; Tables; ; Last updated - 2016-12-02. 2

[13] R. O. Lane, D. A. Nevell, S. D. Hayward, and T. W. Beaney. Maritime anomaly detection and threat assessment. In Information Fusion (FUSION), 2010 13th Conference on, pages 1-8. IEEE, 2010. 2

[14] W. Li, D. Zhang, M. Sun, Y. Yin, and Y. Shen. Loitering detection based on trajectory analysis. In 2015 8th International Conference on Intelligent Computation Technology and Automation (ICICTA), pages 530-533, June 2015. 2

[15] R. Lu, H. Yang, J. Zhu, S. Wu, J. Wang, and D. Bull. Hierarchical video summarization with loitering indication. In 2015 Visual Communications and Image Processing (VCIP), pages 1-4, Dec 2015. 2

[16] S. Mallat. A theory for multiresolution signal decomposition: the wavelet representation. Pattern Analysis and Machine Intelligence, IEEE Transactions on, 11(7):674-693, 1989. 3 
[17] Y. Nam. Loitering detection using an associating pedestrian tracker in crowded scenes. Multimedia Tools and Applications, 74(9):2939-2961, 2015. 2

[18] L. Patino and J. Ferryman. Semantic modelling for behaviour characterisation and threat detection. In The IEEE Conference on Computer Vision and Pattern Recognition (CVPR) Workshops, June 2016. 2, 3

[19] K. Patroumpas, A. Artikis, N. Katzouris, M. Vodas, Y. Theodoridis, and N. Pelekis. Event recognition for maritime surveillance. In EDBTInternational Conference on Extending Database Technology, pages 629-640, 2015. 2

[20] B. Ristic, B. L. Scala, M. Morelande, and N. Gordon. Statistical analysis of motion patterns in ais data: Anomaly detection and motion prediction. In Information Fusion, 2008 11th International Conference on, pages 1-7, June 2008. 1

[21] M. Riveiro, G. Falkman, and T. Ziemke. Improving maritime anomaly detection and situation awareness through interactive visualization. In Information Fusion, 2008 11th International Conference on, pages 1-8, June 2008. 1

[22] G. Rogova and J. Garcia. Contextual knowledge and information fusion for maritime piracy surveillance. Prediction and Recognition of Piracy Efforts Using Collaborative Human-Centric Information Systems, 109:80, 2013. 1

[23] R. Scheepens, N. Willems, H. van de Wetering, G. Andrienko, N. Andrienko, and J. J. van Wijk. Composite density maps for multivariate trajectories. IEEE Transactions on Visualization and Computer Graphics, 17(12):2518-2527, Dec 2011. 1

[24] G. Shafer et al. A mathematical theory of evidence, volume 1. Princeton university press Princeton, 1976. 1 